JOURNAL OF INTEGRAL EQUATIONS

AND APPLICATIONS

Volume 18, Number 1, Spring 2006

\title{
COMPACTNESS ESTIMATES FOR INTEGRAL OPERATORS OF VECTOR FUNCTIONS WITH NONMEASURABLE KERNELS
}

\author{
MARTIN VÄTH \\ Dedicated to Kendall Atkinson
}

\begin{abstract}
Estimates for the measure of noncompactness of integral operators of vector functions are proved. In particular, for linear integral operators of vector functions with nonmeasurable kernels a Mönch type compactness result is obtained.
\end{abstract}

1. Introduction. For scalar functions, it is well known that the Urysohn integral operator

$$
A x(t):=\int_{0}^{1} g(t, s, x(s)) d s, \quad t \in[0,1]
$$

is usually compact. In particular, $A$ is compact in the space $C([0,1])$ under mild continuity assumptions on $g$. This is a consequence of the Arzelá-Ascoli theorem, because the image of bounded sets has equicontinuous norm (provided that, e.g., $g$ is continuous).

Moreover, if $g$ is a Carathéodory function, i.e., $g(\cdot, \cdot, u)$ is (strongly) (Bochner) measurable for each $u$, and $g(t, s, \cdot)$ is continuous for almost all $(t, s) \in[0,1]^{2}$, then, under some growth assumptions on $g$, the operator $A$ maps compactly into the spaces $L_{p}([0,1]), 1 \leq p<\infty$, or, more generally, into regular ideal spaces. These are classical results of Krasnosel'skiı̌ $[\mathbf{9}]$ and Zabreı̌ko, see e.g., $[\mathbf{9}, \mathbf{1 0}, \mathbf{2 8}]$, and it is also possible to weaken the growth conditions slightly $[\mathbf{1 6}, \mathbf{2 0}]$.

We are interested in the case that the functions $x$ and $g$ assume values in Banach (or at least normed) spaces $U$ and $V$, respectively, where the

\footnotetext{
Received by the editors on May 6, 2005 .

Key words and phrases. Integral operator, kernel operator, Urysohn operator, measure of noncompactness, ideal space, spaces of measurable functions.

The paper was written in the framework of a Heisenberg Fellowship (Az. VA 206/1-1 and VA 206/1-2). Financial support by the DFG is gratefully acknowledged.
}

Copyright (C)2006 Rocky Mountain Mathematics Consortium 
integral is understood in the Lebesgue-Bochner sense. In order to prove compactness of $A$, it is natural to assume that $g(t, s, \cdot)$ is a compact function. Since we do not assume completeness of the underlying spaces, let us make precise here that we call a set $M$ in a normed space precompact if its completion its compact, i.e., if each sequence in $M$ contains a Cauchy (not necessarily convergent) subsequence. An operator is called compact if it maps bounded sets into precompact sets.

Concerning vector functions, the compactness of (1) was mainly studied in spaces of continuous functions. If $g(t, s, \cdot)$ is compact, or at least $\gamma$-Lipschitz for some measure $\gamma$ of noncompactness, then, under analogous continuity assumptions on $g$ as in the scalar case, the operator $A$ is compact, respectively $\gamma$-Lipschitz, from $C([0,1], U)$ into $C([0,1], V)$. As one might expect, the proof of this result needs a vector-valued version of the Arzelá-Ascoli theorem (see, e.g., $[\mathbf{1}, \mathbf{6}, \mathbf{1 7}$, 25]). However, the proof is only straightforward if, for each $\|u\| \leq C$, almost all of the values $g(t, s, u)$ belong to the same precompact set (i.e., independent of $t$ and $s$ ). In general, one needs a deep result about the behavior of measures of noncompactness under (Bochner) integration which is essentially due to Mönch $[\mathbf{1 5}]$, see also $[\mathbf{3}, \mathbf{7}, \mathbf{1 1}$, 14] (we will make use of a special case of this result in the proof of Theorem 3). A precise formulation and proof of the above-mentioned compactness result in the space of continuous functions can be found in $[\mathbf{2 4}]$ (see also $[\mathbf{2 6}, \mathbf{2 7}]$ ).

If one wants to avoid the result of Mönch, one can obtain slightly better estimates for some measures of noncompactness, but one has to assume more on the function $g$, e.g., that $g$ is uniformly continuous or at least that $g$ is a strict Carathéodory function [21]. The latter means, by definition, that $(t, s) \mapsto g(t, s, \cdot)$ is (strongly Bochner) measurable as a function from $[0,1]^{2}$ into $C(U, V)$ where $C(U, V)$ is equipped with the topology of uniform convergence on bounded sets. Each strict Carathéodory function is a Carathéodory function, but the converse holds only if $\operatorname{dim} U<\infty$. More precisely, it is necessary (and for separable $U$ also sufficient) for the converse that $g(t, s, \cdot)$ is for almost all $(t, s) \in[0,1]^{2}$ contained in a separable subspace of $C(U, V)$; the latter space is always nonseparable if $\operatorname{dim} U=\infty[\mathbf{2 1}]$. 
Concerning the compactness in regular ideal spaces (like $L_{p}$ ) much less is known about (1). There are also analogous compactness results as in the scalar case, but all these results require in addition that $g$ is a strict Carathéodory function. Although this condition is usually satisfied if each of the maps $g(t, s, \cdot)$ is itself an Urysohn operator [24], this condition is in general rather restrictive, as we have remarked above. On the other hand, an analogous form of the Mönch result holds also for regular ideal spaces [23]. So it is natural to ask whether, using the Mönch result or some generalization, it is possible to drop the requirement that $g$ be a strict Carathéodory function.

The following Theorem 1 destroys all hope in this direction: It shows that such a result is even false in the linear case $g(t, s, u)=k(t, s) u$ where each $k(t, s)$ is an element of the space $\mathcal{L}(U, V)$ of bounded linear operators from $U$ into $V$. Note that such a function $g$ is a Carathéodory function if and only if $k(\cdot, \cdot) u$ is measurable for each $u \in U$. Moreover, $g$ is a strict Carathéodory function if and only if $k: T \times S \rightarrow \mathcal{L}(U, V)$ is measurable. We denote the norm in the spaces $U, V$ and $\mathcal{L}(U, V)$ always by $|\cdot|$.

Theorem 1. Let $T$ and $S$ be measure spaces and $V$ be a nontrivial normed space. Assume that $T$ contains a set of positive finite measure without atoms and that $S$ contains a set of positive finite measure.

Then there exists a separable Banach space $U$ and a map $k: T \times S \rightarrow$ $\mathcal{L}(U, V)$ with $|k(t, s)| \leq 1$ such that $k(\cdot, \cdot) u$ is (strongly Bochner) measurable on $T \times S$ for each $u \in U$ and $k(T \times S)(U)$ is a onedimensional subspace of $V$, and the linear integral operator

$$
K x(t):=\int_{S} k(t, s) x(s) d s, \quad t \in T
$$

acts boundedly from each $L_{p}(S, U), 1 \leq p \leq \infty$, into each $L_{q}(T, V)$, $1 \leq q \leq \infty$, but always fails to be compact. If $S$ has finite measure, it may additionally be arranged that $k(t, s)$ is independent of $s$.

Proof. As a matter of fact, one can always choose $U:=\ell_{1}$. Let $D \subseteq T$ be a set of positive finite measure without atoms. Divide $D$ into two sets $D_{1,1}$ and $D_{1,2}$ of equal measure, and put $E_{1}:=D_{1,1}$; divide $D_{1,1}$, respectively $D_{1,2}$, into two sets $D_{2,1}$ and $D_{2,2}$, respectively 
$D_{2,3}$ and $D_{2,4}$, of equal measure and put $E_{2}:=D_{2,1} \cup D_{2,3}$. Proceed by induction by dividing the sets $D_{n, k}$ analogously and putting $E_{n+1}:=\cup$ $\left\{D_{n+1, k}: k<2^{n+1}\right.$ odd $\}$. Then $E_{n}$ are measurable subsets of $D$ with $\operatorname{mes}\left(E_{n} \Delta E_{m}\right)=\operatorname{mes} D / 2, n \neq m$.

Let $S_{0} \subseteq S$ have positive finite measure (if $S$ has finite measure, put $\left.S_{0}:=S\right)$. Choose $v \in V$ with $|v|=1$ and put $N(t):=\left\{n \in \mathbf{N}: t \in E_{n}\right\}$ and

$$
k(t, s)\left(\xi_{1}, \xi_{2}, \ldots\right):= \begin{cases}\sum_{n \in N(t)} \xi_{n} v & \text { if } s \in S_{0}, \\ 0 & \text { if } s \notin S_{0} .\end{cases}
$$

Then $|k(t, s)| \leq 1$, and since for each fixed $u=\left(\xi_{n}\right)_{n} \in U$ we have

$$
k(t, s) u=\sum_{n=1}^{\infty} \xi_{n} \chi_{E_{n}}(t) \chi_{S_{0}}(s) v
$$

it follows that $k(\cdot, \cdot) u$ is measurable and has its support in the set $D \times$ $S_{0}$ of finite measure. It follows that for each measurable $x$ the function $(t, s) \mapsto k(t, s) x(s)$ is measurable (see, e.g., [19, Theorem A.1.1]), and in view of

$$
|k(t, s) x(s)| \leq \chi_{D}(t) \chi_{S_{0}}(s) \mid x(s)
$$

and the Fubini-Tonelli theorem, $K$ acts from $L_{1}(S, U)$ into $L_{\infty}(T, V)$ and is bounded. Since $D$ and $S_{0}$ have finite measure, the Hölder inequality implies that $K$ acts also from $L_{p}(S, U)$ into $L_{q}(S, V)$ and is bounded.

If $u_{n} \in \ell_{1}$ denotes the $n$th canonical unit vector, we have $k(t, s) u_{n}=$ $\chi_{E_{n}}(t) \chi_{S_{0}}(s) v$. Thus the sequence $x_{n}:=u_{n} \chi_{S_{0}}$ which is bounded in each $L_{p}(S, U)$ is mapped onto the set $\left\{\left(\operatorname{mes} S_{0}\right) \chi_{E_{n}} v: n=1,2, \ldots\right\}$ which fails to be precompact in each $L_{q}(T, V)$, because its elements have the pairwise distance of at least $\left(\right.$ mes $\left.S_{0}\right)(\operatorname{mes} D / 2)^{1 / q}$.

In view of Theorem 1 , it is impossible to say anything about the compactness of (1) in regular ideal spaces without assuming more conditions on the kernel function than just a growth condition (even if the range of the kernel function is bounded and finite-dimensional, and if the Banach spaces involved are separable).

Therefore, it comes as a surprise that the situation is different in the linear case. In fact, it is not accidental that the dual space $U^{*}$ in the above proof is not separable. The next theorem implies that 
for separable $U^{*}$ an analogous result cannot hold. Actually, it is not the separability of $U^{*}$ which is essential for Theorem 2, but the measurability of certain functions, as we will explain later.

In order to formulate our results in sufficient generality, we recall some notions and results from the theory of ideal spaces which can be found, e.g., in $[\mathbf{1 9}]$ (see also $[\mathbf{2 6}, \mathbf{2 7}]$ ).

For simplicity, we assume that all underlying measure spaces are $\sigma$ finite. Thus, let $S$ be a $\sigma$-finite measure space, and let $U$ be a normed space. A normed space $(X,\|\cdot\|)$ of (classes of) measurable functions $x: S \rightarrow U$ is called pre-ideal if for each $x \in X$ and each measurable $y: S \rightarrow U$ the relation $|y(s)| \leq|x(s)|$, for almost all $s \in S$, implies $y \in X$ and $\|y\| \leq\|x\|$. If $X$ is also complete, it is called an ideal space.

To each pre-ideal space $X$ and each normed space $U_{0}$ there corresponds a pre-ideal space $X_{U_{0}}$ consisting of all measurable functions $x: S \rightarrow U_{0}$ with the property that there is some $y \in X$ with $|x(s)|=|y(s)|$ a.e.; we put, of course, $\|x\|_{X_{U_{0}}}:=\|y\|$. In particular, for $U_{0}:=\mathbf{R}$, each pre-ideal space $X$ has a real form $X_{\mathbf{R}}$ of real-valued functions (and vice versa). $X$ is an ideal space if and only if its real form is an ideal space and $U$ is complete.

For a measurable function $x$ and a measurable set $E$, we denote by $P_{E} x$ the function $P_{E} x(s):=\chi_{E}(s) x(s)$. If $E_{n}$ are measurable sets with $E_{1} \supseteq E_{2} \supseteq \cdots$, and $\cap_{n} E_{n}=\varnothing$, we write $E_{n} \downarrow \varnothing$. The notation $E_{n} \uparrow E$ is defined analogously.

The regular part of a pre-ideal space $X$ consists of all functions $x \in X$ with the property that for each sequence $E_{n} \downarrow \varnothing$ we have $\inf _{n}\left\|P_{E_{n}} x\right\|=0$. The regular part is a closed pre-ideal subspace (and thus an ideal space if $X$ is an ideal space). If the regular part is all of $X$, then $X$ is called regular.

We denote the dual space of a normed space $U$ by $U^{*}$. Since $S$ is $\sigma$ finite, the space $X$ has a support $\operatorname{supp} X$, i.e., $\operatorname{supp} X$ is the largest set (determined up to a null set) such that each $x \in X$ vanishes outside $\operatorname{supp} X$. The associate space $X^{\prime}$ of $X$ consists of all measurable functions $y: S \rightarrow U^{*}$ with supp $y \subseteq \operatorname{supp} X$ for which the norm

$$
\|y\|:=\sup _{\|x\|_{X} \leq 1} \int_{S}|y(s)||x(s)| d s
$$


is finite. The associate space is always an ideal space, and we have $\left(X^{\prime}\right)_{\mathbf{R}}=\left(X_{\mathbf{R}}\right)^{\prime}$. For example, for $X=L_{p}$ we have $X^{\prime}=L_{p^{\prime}}$ with $1 / p+1 / p^{\prime}=1$ (even in case $p=\infty$ ). The space $X^{\prime}$ is in a canonical way (by identifying $y$ with an integral functional) isometrically embedded into $X^{*}$. If $U$ has finite dimension, then we have for each regular preideal space $X$ by means of this embedding $X^{\prime}=X^{*}$, but if $X$ fails to be regular or $U$ has infinite dimension, the inclusion can be strict. A special case of our main result can now be formulated as follows.

Theorem 2. Let $U$ be a separable normed space with a separable $U^{*}$, and let $V$ be a Banach space. Let $T$ and $S$ be $\sigma$-finite measure spaces, $X$ be a pre-ideal space of functions $x: S \rightarrow U$ and $Y$ be a pre-ideal space of functions $y: T \rightarrow V$. Let $k: T \times S \rightarrow \mathcal{L}(U, V)$ be such that $k(\cdot, \cdot) u$ is a measurable function for each $u \in U$.

Then the function $|k(\cdot, \cdot)|$ is measurable on $T \times S$, and for the linear integral operators

$$
K x(t):=\int_{S} k(t, s) x(s) d s
$$

and

$$
|K| x(t):=\int_{S}|k(t, s)| x(s) d s
$$

the following holds:

1. If $|K|$ acts from $X_{\mathbf{R}}$ into $Y_{\mathbf{R}}$, then $K$ acts from $X$ into $Y$. Moreover, if $|K|$ is bounded then also $K$ is bounded.

2. Let $|K|$ act compact from $X_{\mathbf{R}}$ into $Y_{\mathbf{R}}$. In addition, suppose that there are sequences $R_{n} \uparrow T \times S$ and precompact sets $P_{n} \subseteq V$ such that for each $x \in B$ and each $n$ there is a measurable function $\lambda_{n, x}: S \rightarrow[0,2]$ satisfying $\lambda_{n, x}(s)=1$ whenever $|x(s)| \leq n$ and

$$
\int_{S} \chi_{R_{n}}(t, s) \lambda_{n, x}(s) k(t, s) x(s) d s \in P_{n}
$$

for almost all $t \in T$. If $Y$ and $X^{\prime}$ are regular, then $K: X \rightarrow Y$ is compact. 
We point out once more that the crucial point of Theorem 2 is that we do not require the measurability of $k: T \times S \rightarrow \mathcal{L}(U, V)$, and therefore none of the classical approaches to prove the compactness of integral operators in $L_{p}$-spaces applies.

If we assume the axiom of choice, the separability of $U^{*}$ implies of course the separability of $U$. However, unless noted otherwise, we will only use the countable axiom of dependent choices for which this fact cannot be proved $[\mathbf{1 2}]$.

Let us now discuss the technical compactness hypothesis (3) for the values of $k(t, s)$. This condition is satisfied if there is a compact set $P$ which is independent of $t$ and $s$ such that $k(t, s)$ maps the unit ball of $U$ into $P$ (put $\lambda_{n, x}:=\chi_{\{s:|x(s)|<n\}}, R_{n}:=T \times S_{n}$ where $S_{n} \uparrow S$ have finite measure, and $P_{n}:=\overline{\operatorname{conv}}\left(n\right.$ mes $\left.\left.\left(S_{n}\right) P \cup\{0\}\right)\right)$.

In addition, the sets $R_{n}$ can be used to disregard small sets of "bad" behavior of $k$, for example, points where $|k(t, s)|$ is large. In particular, if there is a finite-dimensional subspace $P$ which contains the range of $k(t, s)$, then (3) holds (put $R_{n}:=\left\{(t, s) \in T \times S_{n}:|k(t, s)| \leq n\right\}$ with $S_{n}$ and $\lambda_{n, x}$ as above and $P_{n}:=\left\{u \in P:|u| \leq n^{2}\right\}$ ).

The latter shows in particular that Theorem 2 indeed implies that an operator as in Theorem 1 cannot exist if $U^{*}$ is separable.

However, the above remarks can be refined: As a matter of fact, condition (3) is satisfied even if the compact set $P$, respectively the subspace $P$, depends on $s$. This follows from the following theorem which can be considered as a Mönch type compactness result. In this sense, our results can indeed be considered as the proper application of the Mönch technique to integral operators in ideal spaces.

Theorem 3. Assume that there is a measurable function $z: S \rightarrow$ $[0, \infty)$ with $z(s) \neq 0$ for $s \in \operatorname{supp} X$ and a sequence $\widehat{R}_{n} \uparrow T \times S$ such that

$$
B_{n}(s):=\bigcup_{\substack{t \in T \\\left(t, s \in \hat{R}_{n} \\|k(t, s)| \leq n\right.}}\{k(t, s) u:|u| \leq z(s)\}
$$

is precompact for almost all $s$. Then the compactness condition (3) is satisfied with sequences $R_{n} \subseteq \widehat{R}_{n}$, where $R_{n} \uparrow T \times S$, and $\lambda_{n, x}:=$ $\chi_{\{s:|x(s)| \leq n\}}$. 
Corollary 1. The compactness hypothesis concerning (3) holds if at least one of the following two conditions is satisfied.

1. There are precompact sets $P(s) \subseteq V$ such that $k(t, s)$ maps the unit ball of $U$ into $P(s)$ for almost all $(t, s)$.

2. There are finite-dimensional subspaces $P(s) \subseteq V$ such that the range of $k(t, s)$ is contained in $P(s)$ for almost all $(t, s)$.

Proof of Theorem 3. Since $S$ is $\sigma$-finite, there is a sequence $S_{n} \uparrow S$ with mes $S_{n}<\infty$. Now the sequence

$$
R_{n}:=\left\{(t, s) \in \hat{R}_{n}:|k(t, s)| \leq n, s \in S_{n},\right.
$$

$$
\text { and either } z(s) \geq 1 / n \text { or } z(s)=0\}
$$

satisfies $R_{n} \uparrow T \times S$. Theorem 3 is proved if we can show that the set

$$
\begin{aligned}
& P_{n}:=\left\{\int_{S} \chi_{R_{n}}(t, s) \lambda_{n, x}(s) k(t, s) x(s) d s \mid x \in L_{\infty}(S, U),\right. \\
&\left.\|x\|_{\infty} \leq n, \operatorname{supp} x \subseteq \operatorname{supp} z, t \in T\right\}
\end{aligned}
$$

is precompact. Note that, for each $x$, the above integrand is (essentially) bounded by $n^{2} \chi_{S_{n}}$ and thus integrable for almost all $t$ by the Fubini-Tonelli theorem in the form [25, Theorem 1.33]; we understand the definition of $P_{n}$ in the sense that all those pairs $(x, t)$ are omitted for which the integrand is not measurable. To see the precompactness of $P_{n}$, put

$$
\begin{aligned}
C_{n}:=\{y \mid y: S \rightarrow V \text { is measurable with }|y(s)| & \leq n^{2} \chi_{S_{n}}(s) \text { and } \\
y(s) & \left.\in n^{2} B_{n}(s) \cup\{0\}\right\} .
\end{aligned}
$$

Since the functions in $Y_{n}$ are dominated by the integrable function $n^{2} \chi_{S_{n}}$ and since each of the sets $n^{2} B_{n}(s) \cup\{0\}$ is compact, we obtain from the Mönch type theorem [24, Theorem 11.17] (apply part 3 with $f(s, u)=u, M_{n}:=M:=U$, and $\left.r(s) \equiv 0\right)$ that

$$
\left\{\int_{S} y(s) d s: y \in C_{n}\right\}
$$

is precompact. We show that this set contains $P_{n}$. 
Indeed, let some $t \in T$ and a measurable function $x \in L_{\infty}(S, U)$ with $\|x\|_{\infty} \leq n$ and $\operatorname{supp} x \subseteq \operatorname{supp} z$ be given for which the function $y(s):=\chi_{R_{n}}(t, s) \lambda_{n, x}(s) k(t, s) x(s)$ is measurable. The claim follows if we can show that $y$ belongs to $C_{n}$. For almost all $s$ with $y(s) \neq 0$, we have $(t, s) \in R_{n}$, and $|x(s)| \leq n$ which implies $|y(s)| \leq n^{2} \chi_{S_{n}}(s)$. Moreover, for all those $s$, we have $\left|n^{-2} x(s)\right| \leq 1 / n \leq z(s)$, and so $n^{-2} y(s) \in B_{n}(s)$. Hence, $y \in C_{n}$, as required.

2. Proof of the first part of Theorem 2 and auxiliary results. Let us first explain why the separability of $U^{*}$ is assumed for Theorem 2 . The idea of the proof is to consider the associate operator

$$
K^{\prime} y(s):=\int_{T} k(t, s)^{*} y(t) d t
$$

from $Y^{\prime}$ into $X^{\prime}$ where $k(t, s)^{*}$ denotes the adjoint operator of $k(t, s)$. However, since we do not require that $k: T \times S \rightarrow \mathcal{L}(U, V)$ is measurable, it is not true in general that this operator is well-defined even if $|K|$ is bounded from $X_{\mathbf{R}}$ into $Y_{\mathbf{R}}$. In fact, if $U^{*}$ is not separable, it may happen that the function $k(\cdot, \cdot)^{*} f$ may fail to be measurable for $f \in V^{*}$. In particular, this is the case for $k$ as in the proof of Theorem 1 .

This is actually the only place where the separability of $U^{*}$ plays a role (and therefore, we will also formulate our main compactness result without the separability of $U^{*}$ but by assuming that $k(\cdot, \cdot)^{*} f$ is measurable). However, if $U^{*}$ is separable, measurability is no problem as the following result shows.

Proposition 1. Let $R$ be a measure space, $U$ and $V$ be normed spaces, and let $k: R \rightarrow \mathcal{L}(U, V)$ be such that $k(\cdot) u$ is measurable for each $u \in U$. Suppose that $U$ and $U^{*}$ are separable.

Then $|k(\cdot)|$ and $k(\cdot)^{*} f$ are measurable for each $f \in V^{*}$. In particular $(t, f) \mapsto k(t)^{*} f$ is a Carathéodory function.

Proof. Let $\left\{u_{1}, u_{2}, \ldots\right\}$ be a countable dense subset of the unit ball of $U$. Since $\left|k(\cdot) u_{n}\right|$ is measurable for each $n$, it follows that also

$$
|k(\cdot)|=\sup _{n}\left|k(\cdot) u_{n}\right|
$$


is measurable. Moreover, for each $f \in V^{*}$, each $g \in U^{*}$, and each $n$, the function $h_{n}(t):=\left|f\left(k(t) u_{n}\right)-g\left(u_{n}\right)\right|$ is measurable. Since

$$
h(t):=\left|k(t)^{*} f-g\right|=\sup _{n} h_{n}(t),
$$

it follows that also $h$ is measurable. In particular, for each set $E \subseteq R$ of finite measure and each $r>0$ the set $\{t \in E: h(t)<r\}$ belongs to the Lebesgue extension of $R$. By the definition of $h$, we conclude that for each open ball $B \subseteq U^{*}$ the set $\left\{t \in E: k(t)^{*} f \in B\right\}$ belongs to the Lebesgue extension of $R$. Since $U^{*}$ is a separable metric space, its topology has a countable base consisting of open balls. In particular, each open set $O \subseteq U^{*}$ is the countable union of open balls, and so $\left\{t \in E: k(t)^{*} f \in O\right\}$ belongs to the Lebesgue extension of $R$. Since $U^{*}$ is separable, [25, Theorem 1.1] implies that $k(\cdot)^{*} f$ is measurable, as required.

The proof of the following fact is by standard arguments which we recall for the reader's convenience. Only the last statement is apparently new, although not surprising.

Proposition 2. Let $T$ and $S$ be $\sigma$-finite measure spaces, $U$ a normed space, $V$ a Banach space, and let $k: T \times S \rightarrow \mathcal{L}(U, V)$ be such that $k(\cdot, \cdot) u$ and $k(\cdot, \cdot)^{*} f$ are measurable for each $u \in U$ and $f \in V^{*}$, respectively. Assume also that $|k(\cdot, \cdot)|$ is measurable.

If $|K|$ acts from $X_{\mathbf{R}}$ into $Y_{\mathbf{R}}$, then $K$ acts from $X$ into $Y$. If additionally $|K| \in \mathcal{L}\left(X_{\mathbf{R}}, Y_{\mathbf{R}}\right)$, then $K \in \mathcal{L}(X, Y)$ and $K^{\prime} \in \mathcal{L}\left(Y^{\prime}, X^{\prime}\right)$ and also the operator

$$
\left|K^{\prime}\right| y(s):=\int_{T}|k(t, s)| y(t) d t
$$

acts from $Y_{\mathbf{R}}^{\prime}$ into $X_{\mathbf{R}}^{\prime}$ and is bounded with

$$
\left\|\left|K^{\prime}\right|\right\|_{\mathcal{L}\left(Y_{\mathbf{R}}^{\prime}, X_{\mathbf{R}}^{\prime}\right)} \leq\||K|\|_{\mathcal{L}\left(X_{\mathbf{R}}, Y_{\mathbf{R}}\right)} .
$$

Moreover, in this case, $K^{\prime}$ is the restriction of the adjoint operator $K^{*}: Y^{*} \rightarrow X^{*}$ to $Y^{\prime} \subseteq Y^{*}$ (via the canonical identifications).

Proof. Since $(t, s, u) \mapsto k(t, s) u$ is a Carathéodory function, we have for each $x \in X$ that $(t, s) \mapsto k(t, s) x(s)$ is measurable. Since $|K||x|(t)$ 
is finite for almost all $t$, the Fubini-Tonelli theorem in the form $[\mathbf{2 5}$, Theorem 1.33] thus implies that $K x(t)$ is defined for almost all $t$ and that $K x$ is measurable. The obvious estimate $|K x(t)| \leq|K||x|(t)$ implies that $K: X \rightarrow Y$ and that $K$ is bounded if $|K|$ is bounded. If $|K|$ is bounded, we have by Tonelli's theorem that

$$
\begin{aligned}
\sup _{\|y\|_{Y^{\prime}} \leq 1} \sup _{\|x\|_{X} \leq 1} \int_{S} & \int_{T}\left|k(t, s)^{*}\right||y(t)||x(s)| d t d s \\
& =\sup _{\|y\|_{Y^{\prime}} \leq 1} \sup _{\|x\|_{X} \leq 1} \int_{T} \int_{S}|y(t)||k(t, s)||x(s)| d s d t \\
& =\sup _{\|y\|_{Y^{\prime}} \leq 1} \sup _{\|x\|_{X} \leq 1} \int_{T}|y(t)||K||x|(t) d t \leq\||K|\|,
\end{aligned}
$$

which shows that $\left|K^{\prime}\right|$ acts boundedly from $Y_{\mathbf{R}}^{\prime}$ into $X_{\mathbf{R}}^{\prime}$ with (5). Since $(t, s, f) \mapsto k(t, s)^{*} f$ is a Carathéodory function, an analogous argument as above shows that $K^{\prime}$ acts boundedly from $Y^{\prime}$ to $X^{\prime}$. Moreover, by the above calculation, we may apply the Fubini-Tonelli theorem to obtain for each $x \in X$ and each $y \in Y^{\prime}$ (with a similar calculation as above) that

$$
\int_{T} y(t) K x(t) d t=\int_{S} K^{\prime} y(s) x(s) d s
$$

which means that $K^{\prime}$ is the restriction of $K^{*}$.

Remark 1. If $|k|=|k(\cdot, \cdot)|$ fails to be measurable, then Proposition 2 holds anyway, provided we replace $|k|$ in the definition of the operators $|K|$ and $\left|K^{\prime}\right|$ by a measurable function which dominates $|k|$.

For Urysohn operators with strict Carathéodory functions, compactness results have been obtained in [24]. Moreover, even if the kernel function is too singular to generate a compact operator, it is possible to give good estimates for the measure of noncompactness of the image [18]. More precisely, we are interested in estimates for the Hausdorff measure of noncompactness. Recall that for a subset $A$ of a metric space $Z$ the Hausdorff measure of noncompactness is defined as

$$
\chi_{Z}(A):=\inf \{\varepsilon>0: A \text { has a finite } \varepsilon \text {-net in } Z\} .
$$

We will only need a special case of [18] for linear integral operators. This case can be formulated as follows. 
Let $U$ be a normed space, $V$ be a Banach space, and let $S$ and $T$ be $\sigma$-finite measure spaces. Let $X$ and $Y$ be pre-ideal spaces of measurable functions $x: S \rightarrow U$ and $y: S \rightarrow V$, respectively. Let $B \subseteq X$ be bounded, and $k: T \times S \rightarrow \mathcal{L}(U, V)$ be such that $k(\cdot, \cdot) u$ is measurable for each $u \in U$. Besides the linear integral operators $K$ and $|K|$ with kernel functions $k$ and $|k|$, respectively, we consider also the (nonlinear!) operator

$$
|K|_{0} x(t):=\int_{T}|k(t, s) x(s)| d s .
$$

We put

$$
\begin{aligned}
& \gamma_{S}^{0}(k, B):=\sup _{S \supseteq D_{n} \downarrow \varnothing} \limsup _{n \rightarrow \infty} \sup _{x \in B}\left\||K|_{0} P_{D_{n}} x\right\|_{Y_{\mathbf{R}}}, \\
& \gamma_{T}^{0}(k, B):=\sup _{T \supseteq E_{n} \downarrow \varnothing} \limsup _{n \rightarrow \infty} \sup _{x \in B}\left\|P_{E_{n}}|K|_{0} x\right\|_{Y_{\mathbf{R}}} .
\end{aligned}
$$

Theorem 4. Assume in the above situation that $|K| \chi_{E}$ is almost everywhere finite for each $\chi_{E} \in X_{\mathbf{R}}$ with mes $E<\infty$. Suppose that $|K|_{0}$ sends $B$ into the regular part of $Y_{\mathbf{R}}$. Finally, assume that $k(t, s)$ is compact for almost all $(t, s) \in T \times S$ and that $k: T \times S \rightarrow \mathcal{L}(U, V)$ is measurable (which is automatically the case if $\operatorname{dim} U<\infty)$. Then $K$ sends $B$ into the regular part $Y_{0}$ of $Y$, and

$$
\chi_{Y_{0}}(K(B)) \leq \gamma_{S}^{0}(k, B)+\gamma_{T}^{0}(k, B) .
$$

We need also the following convergence result from [18]. We formulate this result only for a special case. In particular, we assume that the limit operator is linear, namely $K$, and that the operator dominating the convergence is $|K|_{0}$. Moreover, we restrict our attention to Carathéodory functions.

Theorem 5. Consider the situation described before Theorem 4. Assume that there are sets $R_{k} \uparrow T \times S$ and Carathéodory functions $g_{n}: T \times S \times U \rightarrow V$ such that $g_{n}(t, s, u) \rightarrow k(t, s) u$ uniformly on each set of the form $R_{k} \times\{u \in U:|u|<k\}$. Assume in addition that

$$
\left|g_{n}(t, s, u)-k(t, s) u\right| \leq|k(t, s) u|
$$


For each natural number $j \leq n$, let $G_{j, n}$ be an operator on $B$ which sends $x \in B$ into a measurable function $G_{j, n} x: T \times S \rightarrow V$ such that

$$
\begin{cases}G_{j, n} x(t, s)=g_{n}(t, s, x(s)) & \text { if }|x(s)|<j \\ \left|G_{j, n} x(t, s)-k(t, s) x(s)\right| \leq|k(t, s) x(s)| & \text { if }|x(s)| \geq j\end{cases}
$$

for almost all $(t, s)$. Put

$$
A_{j, n} x(t):=\int_{S} G_{j, n} x(t, s) d s, \quad t \in T .
$$

Suppose that $|K| \chi_{D}$ is almost everywhere finite for each $\chi_{D} \in X$ with mes $D<\infty$ and that $A_{j, n}: B \rightarrow Y$ and $K: B \rightarrow Y$. Then there are sequences $j_{1}<j_{2}<\cdots$ and $p_{1}<p_{2}<\cdots$ of natural numbers with $j_{n} \leq p_{n}$ and

$$
\limsup _{n \rightarrow \infty} \sup _{x \in B}\left\|A_{j_{n}, p_{n}} x-K x\right\| \leq \gamma_{S}^{0}(k, B)+\gamma_{T}^{0}(k, B) .
$$

3. Measures of noncompactness and a Schauder type theorem. We need also a slight variation of the Hausdorff measure of noncompactness, the so-called inner Hausdorff measure of noncompactness $\chi_{i}$. For a set $A$ in a metric space $Z$, this measure is defined similarly as the Hausdorff measure of noncompactness but with the difference that the $\varepsilon$-net must be chosen as a subset of $A$, i.e.,

$$
\chi_{i}(A):=\chi_{A}(A)
$$

Clearly, $\chi_{Z}(A) \leq \chi_{i}(A)$. With this measure, and the Hausdorff measure of noncompactness, we can prove the following quantitative version of the Schauder theorem.

Theorem 6. Let $X$ and $Y$ be normed linear spaces, and let $K \in$ $\mathcal{L}(X, Y)$. Let $B \subseteq X$ and $B_{*} \subseteq Y^{*}$ be bounded, and let $M:=B-B$ and $C:=\sup \{\|x\|: x \in M\}$. Let $c>0$ and $\rho>0$ satisfy

$$
\sup _{f \in B_{*}}|f(y)| \geq c\|y\|_{Y}, \quad y \in K(M),\|y\|_{Y}>\rho .
$$


Then

$$
\chi_{Y}(K(B)) \leq \chi_{i}(K(B)) \leq \max \left\{\frac{C}{c} \chi_{X^{*}}\left(K^{*}\left(B_{*}\right)\right), \rho\right\} .
$$

If we assume the axiom of choice, then the Hahn-Banach theorem holds and implies that (11) is true with $c=1$ and all $\rho>0$ when $B_{*}$ is the unit ball of $Y^{*}$. Therefore, a special case of Theorem 6 is the following quantitative variant of the Schauder theorem (assuming the axiom of choice):

Corollary 2. Let $X$ and $Y$ be normed linear spaces, and let $K \in \mathcal{L}(X, Y)$. If $B(X)$ and $B\left(Y^{*}\right)$ denote the corresponding unit balls, then

$$
\chi_{i}(K(B(X))) \leq 2 \chi\left(K^{*}\left(B\left(Y^{*}\right)\right)\right) .
$$

In particular, $K$ is compact if $K^{*}$ is compact.

Proof of Theorem 6. Given $\delta>\chi_{X^{*}}\left(K^{*}\left(B_{*}\right)\right)$, we find a finite $\delta$ net $\left\{g_{1}, \ldots, g_{n}\right\} \subseteq X^{*}$ for $K^{*}\left(B_{*}\right)$. Define a bounded linear function $h: X \rightarrow \mathbf{K}^{n}(\mathbf{K}=\mathbf{R}$ or $\mathbf{K}=\mathbf{C})$ by

$$
h(x):=\left(g_{1}(x), \ldots, g_{n}(x)\right) .
$$

Endowing $\mathbf{K}^{n}$ with the max-norm, we have that the image $h(B) \subseteq \mathbf{K}^{n}$ is bounded and thus $\chi_{i}(h(B))=0$. Hence, we find for each $\varepsilon>0$, a finite set $N:=\left\{x_{1}, \ldots, x_{m}\right\} \subseteq B$ such that $h(N)$ is an $\varepsilon$-net for $h(B)$. We claim that $K(N)$ is a $\max \{(C \delta+\varepsilon) / c, \rho\}$-net for $K(B)$.

In fact, given $x \in B$, choose $k$ such that $\left\|h(x)-h\left(x_{k}\right)\right\|<\varepsilon$. For each $f \in B_{*}$ we have

$$
f\left(K x-K x_{k}\right)=f K\left(x-x_{k}\right)=\left(K^{*} f\right)\left(x-x_{k}\right) .
$$

Choose some $j$ with $\left\|K^{*} f-g_{j}\right\|_{X^{*}} \leq \delta$. Then

$$
\begin{aligned}
\left\|f\left(K x-K x_{k}\right)\right\| & =\left\|\left(K^{*} f\right)\left(x-x_{k}\right)\right\| \\
& =\left\|g_{j}\left(x-x_{k}\right)+\left(K^{*} f-g_{j}\right)\left(x-x_{k}\right)\right\| \\
& \leq\left\|g_{j}(x)-g_{j}\left(x_{k}\right)\right\|+\left\|K^{*} f-g_{j}\right\|\left\|x-x_{k}\right\| \\
& \leq\left\|h(x)-h\left(x_{k}\right)\right\|+\delta \cdot C \leq C \delta+\varepsilon,
\end{aligned}
$$


and so (11) implies that either $\left\|K x-K x_{k}\right\|_{Y} \leq \rho$ or $\left\|K x-K x_{k}\right\|_{Y} \leq$ $(C \delta+\varepsilon) / c$, as required.

4. Formulation of the main results. Before we formulate our main theorem, we need another notion about a pre-ideal space $X: X$ is called $\beta$-almost perfect if $X_{\mathbf{R}}$ has the property that the relations $0 \leq x_{n}(s) \uparrow x(s)$ a.e. and $x \in X_{\mathbf{R}}$ imply that $\|x\| \leq \beta \sup _{n}\left\|x_{n}\right\|$. Each regular pre-ideal space is automatically $\beta$-almost perfect with $\beta=1$ by [19, Corollary 3.3.4].

We point out once more that the measurability assumption for $k(\cdot, \cdot)^{*} f$ and $|k(\cdot, \cdot)|$ in the following result are automatically satisfied by Proposition 1 if $U$ and $U^{*}$ are separable.

Theorem 7. Let $U$ be a normed space, and let $V$ be a Banach space. Let $T$ and $S$ be $\sigma$-finite measure spaces, $X$ a pre-ideal space of functions $x: S \rightarrow U$ and $Y$ a pre-ideal space of functions $y: T \rightarrow V$. Let $k: T \times S \rightarrow \mathcal{L}(U, V)$ be such that the functions $k(\cdot, \cdot) u, k(\cdot, \cdot)^{*} f$, and $|k(\cdot, \cdot)|$ are measurable for each $u \in U$ and each $f \in V^{*}$.

Assume that $|K| \in \mathcal{L}\left(X_{\mathbf{R}}, Y_{\mathbf{R}}\right)$ and, consequently, the nonlinear operator $|K|_{0}$ from (6) is bounded and $K \in \mathcal{L}(X, Y)$. Then also the operator $\left|K^{\prime}\right|$ in Proposition 2 is bounded from $Y_{\mathbf{R}}^{\prime}$ into $X_{\mathbf{R}}^{\prime}$, and so for bounded $B \subseteq X$ the numbers

$$
\begin{aligned}
\gamma_{S}^{0}(k, B) & :=\sup _{S \supseteq D_{n} \downarrow \varnothing} \limsup _{n \rightarrow \infty} \sup _{x \in B}\left\||K|_{0} P_{D_{n}} x\right\|_{Y_{\mathbf{R}}}, \\
\gamma_{T}^{0}(k, B) & :=\sup _{T \supseteq E_{n} \downarrow \varnothing} \limsup _{n \rightarrow \infty} \sup _{x \in B}\left\|P_{E_{n}}|K|_{0} x\right\|_{Y_{\mathbf{R}}}, \\
\gamma_{S}^{\prime}(k) & :=\sup _{S \supseteq D_{n} \downarrow \varnothing} \limsup _{n \rightarrow \infty}\left\|P_{D_{n}}\left|K^{\prime}\right|\right\|_{\mathcal{L}\left(Y_{\mathbf{R}}^{\prime}, X_{\mathbf{R}}^{\prime}\right)}, \\
\gamma_{T}^{\prime}(k) & :=\sup _{T \supseteq E_{n} \downarrow \varnothing} \limsup _{n \rightarrow \infty}\left\|\left|K^{\prime}\right| P_{E_{n}}\right\|_{\mathcal{L}\left(Y_{\mathbf{R}}^{\prime}, X_{\mathbf{R}}^{\prime}\right)}
\end{aligned}
$$

are finite. Let $Y$ be $\beta$-almost perfect and assume that $\left|K^{\prime}\right|\left(Y_{\mathbf{R}}^{\prime}\right)$ is contained in the regular part of $X_{\mathbf{R}}^{\prime}$. Moreover, assume that the compactness assumption (3) for the values of $k$ is satisfied. Then we have for $C:=\sup \{\|x-y\|: x, y \in B\}$ that

$$
\chi_{Y}(K(B)) \leq \gamma_{S}^{0}(k, B)+\gamma_{T}^{0}(k, B)+C \beta\left(\gamma_{S}^{\prime}(k)+\gamma_{T}^{\prime}(k)\right) .
$$


If $\chi_{\text {supp } K(B)} \in Y_{\mathbf{R}}^{\prime \prime}$ and if there is a precompact set $P \subseteq V$ such that $K x(t) \in P$ a.e. for each $x \in B$, then we even have the stronger estimate

$$
\chi_{i}(K(B)) \leq C \beta\left(\gamma_{S}^{\prime}(k)+\gamma_{T}^{\prime}(k)\right) .
$$

For the case that $B$ is the unit ball of $X$, it is not necessary to calculate the quantities $\gamma_{S}^{\prime}(k)$ and $\gamma_{T}^{\prime}(k)$. More precisely, we obtain for the quantities

$$
\begin{aligned}
\gamma_{S}(k):=\sup _{S \supseteq D_{n} \downarrow \varnothing} \limsup _{n \rightarrow \infty}\left\||K| P_{D_{n}}\right\|_{\mathcal{L}\left(X_{\mathbf{R}}, Y_{\mathbf{R}}\right)}, \\
\gamma_{T}(k):=\sup _{T \supseteq E_{n} \downarrow \varnothing} \limsup _{n \rightarrow \infty}\left\|P_{E_{n}}|K|\right\|_{\mathcal{L}\left(X_{\mathbf{R}}, Y_{\mathbf{R}}\right)}
\end{aligned}
$$

the following consequence:

Corollary 3. Let all assumptions of Theorem 7 be satisfied with $B:=\{x \in X:\|x\| \leq 1\}$. Then the conclusion of Theorem 7 holds with the estimates

$$
\begin{aligned}
\chi_{Y}(K(B)) & \leq\left(\gamma_{S}^{0}(k, B)+\gamma_{T}^{0}(k, B)\right)+2 \beta\left(\gamma_{S}(k)+\gamma_{T}(k)\right) \\
& \leq(1+2 \beta)\left(\gamma_{S}(k)+\gamma_{T}(k)\right)
\end{aligned}
$$

and

$$
\chi_{i}(K(B)) \leq 2 \beta\left(\gamma_{S}(k)+\gamma_{T}(k)\right),
$$

respectively.

If we assume in Corollary 3 that $X^{\prime}$ is regular, then we do not have to consider $\left|K^{\prime}\right|$ at all, because the assumption that $\left|K^{\prime}\right|$ sends $Y^{\prime}$ into the regular part of $X^{\prime}$ is then automatically satisfied by Proposition 2 . We recall once more that each regular pre-ideal space is automatically $\beta$-almost perfect with $\beta=1$.

Corollary 4. Let all assumptions of Theorem 7 be satisfied with $B:=\{x \in X:\|x\| \leq 1\}$ and assume that $Y$ is regular. Then the following statements are equivalent:

1. $|K|$ is compact. 
2. For each sequence $T \supseteq E_{n} \downarrow \varnothing$ we have $\left\|P_{E_{n}}|K|\right\|_{\mathcal{L}\left(X_{\mathbf{R}}, Y_{\mathbf{R}}\right)} \rightarrow 0$.

3. For each sequence $S \supseteq D_{n} \downarrow \varnothing$ we have $\left\||K| P_{D_{n}}\right\|_{\mathcal{L}\left(X_{\mathbf{R}}, Y_{\mathbf{R}}\right)} \rightarrow 0$.

4. For each of the sequences $T \supseteq E_{n} \downarrow \varnothing$ and $S \supseteq D_{n} \downarrow \varnothing$, we have $\left\|P_{E_{n}}|K| P_{D_{n}}\right\|_{\mathcal{L}\left(X_{\mathbf{R}}, Y_{\mathbf{R}}\right)} \rightarrow 0$.

If one of these statements is true, then $K$ is compact.

Theorem 2 follows from Corollary 4 (and our earlier considerations).

Remark 2. If $|k(\cdot, \cdot)|$ fails to be measurable, then a result analogous to Theorem 7 and the above corollaries holds true, provided that we replace $|k|$ in the definition of $|K|$ and $\left|K^{\prime}\right|$ by a measurable function which dominates $|k|$.

Remark 3. In our proof of Theorem 7 (and its corollaries, including Theorem 2), we make use of the Hahn-Banach extension theorem which requires the axiom of choice. However, Theorem 7 (and its corollaries) remains true also if we reject the axiom of choice and assume only the (countable) axiom of dependent choices [8] under the following minor restrictions:

1. Since we cannot make use of the Hahn-Banach theorem, we must require that $V$ has the bidual property

$$
|v|=\sup _{|f|_{V^{*} \leq 1}}|f(v)|, \quad v \in V .
$$

Even if one rejects the (general) axiom of choice, this property can be proved for a very large class of spaces, including all separable spaces $V$ [5, p. 183]. If $V$ does not satisfy the bidual property but at least

$$
|v| \leq \alpha \sup _{|f|_{V^{*}} \leq 1}|f(v)|, \quad v \in V
$$

with some constant $\alpha \in[1, \infty)$ then Theorem 7 (and its corollaries) remain true, provided that $Y$ is $(\beta / \alpha)$-almost perfect. Note that, without the axiom of choice, it is consistent to assume that for any $\alpha_{0} \geq 1$ there are spaces satisfying (14) with $\alpha \geq \alpha_{0}$ but not with any smaller number [4]. In fact, each ideal space which is $\alpha$-almost perfect only for $\alpha \geq \alpha_{0}$ can be assumed to have this property $[\mathbf{1 9}, \mathbf{2 2}]$. 
2. For the compactness condition (3) we require not only that for each $n$ and $x$ a corresponding function $\lambda_{n, x}$ exists, but we assume also that $(n, x) \mapsto \lambda_{n, x}$ is a function (in the sense of the underlying set theory). This is of course automatically the case if we assume the axiom of choice, and this is also the case if $\lambda_{n, x}$ can be given constructively as in Theorem 3 .

5. Proof of the main results. Let us first show why Theorem 7 implies Corollaries 3 and 4.

Proof of Corollary 3. Applying Proposition 2 to the integral operator with kernel $(t, s) \mapsto\left|\chi_{E}(t) k(t, s)\right|$, we obtain

$$
\left\|\left|K^{\prime}\right| P_{E}\right\| \leq\left\|P_{E}|K|\right\|
$$

Similarly, we find with the kernel $(t, s) \mapsto\left|\chi_{D}(s) k(t, s)\right|$ that

$$
\left\|P_{D}\left|K^{\prime}\right|\right\| \leq\left\||K| P_{D}\right\|
$$

These estimates show that $\gamma_{S}^{\prime}(k) \leq \gamma_{S}(k)$ and $\gamma_{T}^{\prime}(k) \leq \gamma_{T}(k)$, and the claim follows by Theorem 7 .

Proof of Corollary 4. The equivalence of the four statements is a standard result for scalar linear integral operators, see e.g., [2, 13]. If these four statements are true, we have in particular $\gamma_{S}(k)=\gamma_{T}(k)=0$, and so the compactness of $K$ follows from Corollary 3 .

Proof of Theorem 7. The claim about $\left|K^{\prime}\right|$ follows from Proposition 2. We first show (13). Thus, let $P \subseteq V$ be precompact and contain $K x(t)$ almost everywhere for each $x \in B$, and suppose that $\chi_{E} \in Y_{\mathbf{R}}^{\prime \prime}$ with $E:=\operatorname{supp} K(B)$. Since $V$ is a Banach space, the set $\bar{P}$ is compact, and thus also $P_{0}:=\bar{P}-\bar{P}$ is compact.

Let $c_{0} \in\left(0, \alpha^{-1}\right)$, with $\alpha$ from $(14), c_{1} \in(0,1)$, and $\rho_{0}>0$ arbitrary. By (14), we find for each nonzero point of $P_{0}$ some $f \in V^{*}$ with $|f|=1$ such that the relation $|f(v)| \geq c_{0}|v|$ holds for all $v$ in a neighborhood of this point. By the compactness of $\left\{v \in P_{0}:|v| \geq \rho_{0}\right\}$, we find (by considering a finite set of corresponding $f$ 's) a finite-dimensional 
subspace $V^{\prime} \subseteq V^{*}$ such that

$$
\sup _{\substack{f \in V^{\prime} \\|f| \leq 1}}|f(v)| \geq c_{0}|v|, \quad v \in P_{0},|v| \geq \rho_{0}
$$

If we denote by $i: V \rightarrow\left(V^{\prime}\right)^{*}$ the canonical (evaluation) embedding, (15) can be read as

$$
|i(v)| \geq c_{0}|v|, \quad v \in P_{0},|v| \geq \rho_{0} .
$$

Let now $M:=B-B$, and let $B_{*}$ denote the subset of all functions of the unit ball of $Y^{\prime}$ which attain their values in $V^{\prime}$, i.e., $B_{*}$ denotes the unit ball of $\left(Y^{\prime}\right)_{V^{\prime}}$. We claim that (16) implies

$$
\sup _{z \in B_{*}}\left|\int_{T} z(t) y(t) d t\right| \geq c_{0} \beta^{-1}\|y\|_{Y}-c_{0} \rho_{0}\left\|\chi_{E}\right\|_{Y_{\mathbf{R}}^{\prime \prime}}, \quad y \in K(M)
$$

To see this, let $y \in K(M)$ be given. Since $y \in K(B)-K(B)$, we have almost everywhere $y(t) \in P-P \subseteq P_{0}$. From (16), we obtain $|i(y(t))|+c_{0} \rho_{0} \geq c_{0}|y(t)|$. Since $y(t)=0$ for almost all $t \notin E$, we have even $|i(y(t))|+c_{0} \rho_{0} \chi_{E}(t) \geq c_{0}|y(t)|$ which implies, since $Y \subseteq Y^{\prime \prime}$ in a canonical way,

$$
\||i \circ y|\|_{Y_{\mathbf{R}}^{\prime \prime}} \geq c_{0}\||y|\|_{Y_{\mathbf{R}}^{\prime \prime}}-c_{0} \rho_{0}\left\|\chi_{E}\right\|_{Y_{\mathbf{R}}^{\prime \prime}}
$$

Since $Y$ is $\beta$-almost perfect, we have by [19, Corollary 3.4.4] the estimate $\||y|\|_{Y_{\mathbf{R}}^{\prime \prime}} \geq \beta^{-1}\||y|\|_{Y_{\mathbf{R}}}$, and so

$$
\||i \circ y|\|_{Y_{\mathbf{R}}^{\prime \prime}} \geq c_{0} \beta^{-1}\|y\|_{Y}-c_{0} \rho_{0}\left\|\chi_{E}\right\|_{Y_{\mathbf{R}}^{\prime \prime}}
$$

Since $V^{\prime}$ has finite dimension, it is reflexive, and so we assume that it is the dual of $W \cong\left(V^{\prime}\right)^{*}$. By [19, Theorem 3.4.4], we have

$$
\begin{aligned}
\sup _{z \in B_{*}}\left|\int_{T} z(t) y(t) d t\right| & =\sup _{z \in B_{*}}\left|\int_{T} i(y(t)) z(t) d t\right| \\
& =\|i \circ y\|_{\left(Y_{V^{\prime}}^{\prime}\right)^{\prime}}=\|i \circ y\|_{\left(Y_{W}\right)^{\prime \prime}}=\||i \circ y|\|_{Y_{\mathbf{R}}^{\prime \prime}}
\end{aligned}
$$

Combining our previous formulas, we thus have proved (17). 
Identifying $B_{*} \subseteq Y^{\prime}$ with a subset of $Y^{*}$, and putting $b:=\left\|\chi_{E}\right\|_{Y_{\mathbf{R}}^{\prime \prime}}$, we can read (17) as

$$
\sup _{f \in B_{*}}|f(y)| \geq c_{0} \beta^{-1}\|y\|_{Y}-c_{0} \rho_{0} b, \quad y \in K(M) .
$$

For $|y| \geq \rho:=\beta \rho_{0} b /\left(1-c_{1}\right)$, we have in particular

$$
\begin{gathered}
\sup _{f \in B_{*}}|f(y)| \geq c_{0} c_{1} \beta^{-1}\|y\|_{Y} \\
+c_{0}\left(1-c_{1}\right) \beta^{-1}\|y\|_{Y}-c_{0} \rho_{0} b \geq c_{0} c_{1} \beta^{-1}\|y\|_{Y} \\
\|y\|_{Y} \geq \rho .
\end{gathered}
$$

Theorem 6 thus implies that

$$
\chi_{i}(K(B)) \leq \max \left\{\frac{C \beta}{c_{0} c_{1}} \chi_{X^{*}}\left(K^{*}\left(B_{*}\right)\right), \rho\right\} .
$$

The crucial point is now that the space $V^{\prime}$ is locally compact and separable (because $\operatorname{dim} V^{\prime}<\infty$ ). This implies that the Carathéodory function $(t, s, f) \mapsto k(t, s)^{*} f$ is actually a strict Carathéodory function by $[\mathbf{2 4}$, Theorem 8.15$]$, i.e., $k(\cdot, \cdot)^{*}$ is measurable as a function from $T \times S$ into $\mathcal{L}\left(V^{\prime}, U^{*}\right)$, and so formula (9) applies for the restriction $K^{\prime}: Y_{V^{\prime}}^{\prime} \rightarrow X^{\prime}$. Since $B_{*} \subseteq Y_{V^{\prime}}^{\prime}$ and $K^{\prime}$ is the restriction of the adjoint operator (Proposition 2), we thus have

$$
\chi_{X^{*}}\left(K^{*}\left(B_{*}\right)\right) \leq \chi_{X_{0}^{\prime}}\left(K^{\prime}\left(B_{*}\right)\right) \leq \gamma_{S}^{\prime}(k)+\gamma_{T}^{\prime}(k) .
$$

Combining our previous formulas, we find

$$
\chi_{i}(K(B)) \leq \max \left\{\frac{C \beta}{c_{0} c_{1}}\left(\gamma_{S}^{\prime}(k)+\gamma_{T}^{\prime}(k)\right), \frac{\beta \rho_{0} b}{1-c_{1}}\right\} .
$$

Since $c_{0} \in\left(0, \alpha^{-1}\right), c_{1} \in(0,1)$, and $\rho_{0}>0$ were arbitrary, we obtain (13) (with $\beta$ replaced by $\alpha \beta$ ).

In order to prove (12), we assume without loss of generality that supp $Y=T$. By [19, Corollary 2.2.7], we find then a sequence of sets $T_{n} \uparrow T$ with $\chi_{T_{n}} \in Y_{\mathbf{R}}$. Choose $R_{n}, P_{n}$ and $\lambda_{n, x}$ corresponding to assumption (3). Replacing $R_{n}$ by its intersection with $T_{n} \times S$ if necessary, we may assume that $R_{n} \subseteq T_{n} \times S$. 
We apply Theorem 5 with $g_{n}(t, s, u):=P_{R_{n}} k(t, s) u$. Since $R_{1} \subseteq R_{2} \subseteq$ $\cdots$, we have $g_{n}(t, s, u) \rightarrow k(t, s) u$ uniformly on each set $R_{k} \times U$. Moreover,

$$
\left|g_{n}(t, s, u)-k(t, s) u\right| \leq|k(t, s) u| .
$$

For natural numbers $j \leq n$ and $x \in B$, put

$$
G_{j, n} x(t, s):=\lambda_{n, x}(s) g_{n}(t, s, x(s))=\lambda_{n, x}(s) P_{R_{n}} k(t, s) x(s)
$$

and note that, in view of $\lambda_{n, x}(s) \in[0,2]$,

$$
\left|G_{j, n} x(t, s)-k(t, s) x(s)\right| \leq|k(t, s) x(s)| .
$$

Moreover, if $|x(s)|<j \leq n$, then $\lambda_{n, x}(s)=1$, and so $G_{j, n} x(t, s)=$ $g_{n}(t, s, x(s))$. By Theorem 5 , we thus find for each $\varepsilon>0$ indices $j \leq n$ with

$$
\sup _{x \in B}\left\|A_{j, n} x-K x\right\| \leq \gamma_{S}^{0}(k, B)+\gamma_{T}^{0}(k, B)+\varepsilon .
$$

Let $K_{n}$ denote the linear integral operator with kernel $k_{n}(t, s) u:=$ $g_{n}(t, s, u)$, and put $D_{n} x:=\lambda_{n^{2}, x}$. By the linearity of $k_{n}(t, s)$, we have $A_{j, n} x=K_{n} D_{n} x$. Condition (3) implies that for each $x \in B$ the values $K_{n} D_{n} x(t)$ are for almost all $t$ contained in a precompact set. Moreover, the support of the image $\left(K_{n} D_{n}\right)(B)$ is contained in $T_{n}$. Hence, observing that $\left|k_{n}\right| \leq|k|$, we can apply (13) with the operator $K_{n}$ and the set $B_{n}:=D_{n} B$ and obtain

$$
\begin{aligned}
\left.\chi_{i}\left(A_{j, n}\right)(B)\right) & =\chi_{i}\left(K_{n}\left(B_{n}\right)\right) \leq C \beta\left(\gamma_{S}^{\prime}\left(k_{n}\right)+\gamma_{T}^{\prime}\left(k_{n}\right)\right) \\
& \leq C \beta\left(\gamma_{S}^{\prime}(k)+\gamma_{T}^{\prime}(k)\right) .
\end{aligned}
$$

In particular, for each $\varepsilon_{0}>C \beta\left(\gamma_{S}^{\prime}(k)+\gamma_{T}^{\prime}(k)\right)$, we find a finite $\varepsilon_{0}$-net for $A_{j, n}(B)$ which by (18) must be a finite $\left(\varepsilon_{0}+\gamma_{S}^{0}(k, B)+\gamma_{T}^{0}(k, B)+\varepsilon\right)$ net for $K(B)$. Hence, $\chi_{Y}(K(B)) \leq \varepsilon_{0}+\gamma_{S}^{0}(k, B)+\gamma_{T}^{0}(k, B)+\varepsilon$, and (12) follows.

\section{REFERENCES}

1. A. Ambrosetti, Un teorema di esistenza per le equazioni differenziali negli spazi di Banach, Rend. Sem. Mat. Univ. Padova 39 (1967), 349-361. 
2. J. Appell and M. Väth, Misure di non compattezza di insiemi ed operatori integrali in spazi di funzioni misurabili, Rend. Mat. Appl. (7) 21 (2001), 159-190.

3. J. Appell, M. Väth and A. Vignoli, Compactness and existence results for ordinary differential equations in Banach spaces, Z. Anal. Anwendungen 18 (1999), $569-584$.

4. G. Dirr and M. Väth, Continuity of near-duality maps and characterizations of ideal spaces of measurable functions, in Recent trends in nonlinear analysis (J. Appell, ed.), (Festschrift dedicated to Alfonso Vignoli on the Occasion of His Sixtieth Birthday), Birkhäuser, Berlin, 2000, pp. 139-148.

5. H.G. Garnir, M. de Wilde and J. Schmets, Analyse fonctionnelle. Théorie constructive des espaces linéaires à semi-normes, tome I: Théorie générale, Birkhäuser, Basel, 1968.

6. H.-P. Heinz, Theorems of Ascoli type involving measures of noncompactness, Nonlinear Anal. 5 (1981), 277-286.

7. - On the behaviour of measures of noncompactness with respect to differentiation and integration of vector-valued functions, Nonlinear Anal. 7 (1983), 1351-1371.

8. T.J. Jech, The axiom of choice, North-Holland Publ. Company, Amsterdam, 1973.

9. M.A. Krasnoselskiı̆, Topological methods in the theory of nonlinear integral equations, Gostehizdat, Moscow, 1956 (Russian); Pergamon Press, Oxford, 1964 (English).

10. M.A. Krasnoselskiı̌, P.P. Zabreı̌ko, E.I. Pustylnik and P.E. Sobolevskiü, Integral operators in spaces of summable functions, Nauka, Moscow, 1958 (Russian); Noordhoff, Leyden, 1976 (English).

11. M. Kunze and G. Schlüchtermann, Strongly generated Banach spaces and measures of noncompactness, Math. Nachr. 191 (1998), 197-214.

12. W.A.J. Luxemburg and M. Väth, The existence of non-trivial bounded functionals implies the Hahn-Banach extension theorem, Z. Anal. Anwendungen 20 (2001), 267-279.

13. W.A.J. Luxemburg and A.C. Zaanen, Compactness of integral operators in Banach function spaces, Math. Ann. 149 (1963), 150-180.

14. H. Mönch, Boundary value problems for nonlinear ordinary differential equations in Banach spaces, Nonlinear Anal. 4 (1980), 985-999.

15. H. Mönch and G.-F. von Harten, On the Cauchy problem for ordinary differential equations in Banach spaces, Arch. Math. (Basel) 39 (1982), 153-160.

16. T.K. Nurekenov, Conditions for complete continuity of an Uryson integral operator, Dokl. Akad. Nauk SSSR 321 (1991), 905-909 (Russian); Soviet Math. Dokl. 44 (1992), 830-835 (English).

17. R.D. Nussbaum, A generalization of the Ascoli theorem and an application to functional differential equations, J. Math. Anal. Appl. 35 (1971), 600-610.

18. M. Väth, Convergence theorems and measures of noncompactness for noncompact Urysohn operators in ideal spaces, J. Integral Equations Appl., to appear.

19. — Ideal spaces, Lecture Notes in Math., vol. 1664, Springer, Berlin, 1997. 
20. M. Väth, Approximation, complete continuity, and uniform measurability of the Uryson operator on general measure spaces, Nonlinear Anal. 33 (1998), 715-728.

21. Complete continuity of some nonlinear Volterra operator in Banach spaces, Z. Anal. Anwendungen 17 (1998), 23-35.

22. - The dual space of $L_{\infty}$ is $L_{1}$, Indag. Math. 9 (1998), 619-625.

23. - A compactness criterion of mixed Krasnoselskiu-Riesz type in regular ideal spaces of vector functions, Z. Anal. Anwendungen 18 (1999), 713-732.

24. - Volterra and integral equations of vector functions, Marcel Dekker, New York, 2000.

25. - Integration theory. A second course, World Scientific Publ., Singapore, 2002.

26. A.C. Zaanen, Integration, North-Holland Publ. Co., Amsterdam, 1967.

27. P.P. Zabreı̌ko, Ideal spaces of functions I, Vestnik Jaroslav. Univ. 8 (1974), 12-52 (in Russian).

28. P.P. Zabreı̌ko, A.I. Koshelev, M.A. Krasnosel'skiı̌, S.G. Mikhlin, L.S. Rakovshchik and V.Ya. Stet'senko, Integral equations - a reference text, Noordhoff, Leyden, 1975.

Universität Würzburg, Math. Institut, Am Hubland, 97074 Würzburg, GERMANY

E-mail address: vaeth@mathematik.uni-wuerzburg.de 